\title{
Multivariate Classification of Blood Oxygen Level-Dependent fMRI Data with Diagnostic Intention: A Clinical Perspective
}

\author{
B. Sundermann, D. Herr, W. Schwindt, and B. Pfleiderer
}

\begin{abstract}
SUMMARY: There has been a recent upsurge of reports about applications of pattern-recognition techniques from the field of machine learning to functional MR imaging data as a diagnostic tool for systemic brain disease or psychiatric disorders. Entities studied include depression, schizophrenia, attention deficit hyperactivity disorder, and neurodegenerative disorders like Alzheimer dementia. We review these recent studies which—despite the optimism from some articles_-predominantly constitute explorative efforts at the proof-ofconcept level. There is some evidence that, in particular, support vector machines seem to be promising. However, the field is still far from real clinical application, and much work has to be done regarding data preprocessing, model optimization, and validation. Reporting standards are proposed to facilitate future meta-analyses or systematic reviews.
\end{abstract}

ABBREVIATIONS: $\mathrm{ADHD}=$ attention deficit hyperactivity disorder; $\mathrm{CV}=$ cross-validation; $\mathrm{LDA}=$ linear discriminant analysis; MVPA $=$ multivariate pattern analysis; SVM = support vector machine

$F$ unctional MR imaging based on blood oxygen level-dependent signal changes that are measured by using fast $\mathrm{T} 2^{*}$-sensitive echo-planar imaging techniques provides an indirect measure of neural activity in the brain. It has an enormous impact on basic research in the field of cognitive neurosciences ${ }^{1}$ and has been applied in numerous group studies with the aim of clarifying disease mechanisms in psychiatric and neurologic disorders, some of which do not exhibit obvious structural alterations (eg, Zhang and Raichle ${ }^{2}$ and Chen et $\mathrm{al}^{3}$ ). However, the applicability of fMRI to single subjects in clinical settings has been limited to a few indications, mainly in the context of surgery planning. ${ }^{4,5}$

Although there has been a substantial effort to identify neuroimaging biomarkers for psychiatric disorders ${ }^{6,7}$ (eg, schizophrenia, ${ }^{8}$ depression, ${ }^{9}$ and neurodegenerative disorders like Alzheimer dementia ${ }^{10}$ with the goal of including biomarkers in official diagnostic criteria, ${ }^{11}$ ), to date capturing functional aspects in diagnostic imaging is almost limited to tracer studies in certain kinds of neurodegeneration. ${ }^{6,12,13}$ In clinical practice, neuroradiologic MR imaging examinations are broadly confined to the

Received May 31, 2013; accepted after revision June 19

From the Department of Clinical Radiology (B.S., W.S., B.P.), University Hospital Münster, Münster, Germany; and Department of Psychiatry and Psychotherapy (D.H.), University of Cologne, Cologne, Germany.

Please address correspondence to Benedikt Sundermann, MD, Department of Clinical Radiology, University Hospital Münster, Albert-Schweitzer-Campus 1,

Gebäude A1, 48149 Münster, Germany; e-mail: benedikt.sundermann@ukmuenster.de

三 Indicates article with supplemental on-line tables

http://dx.doi.org/10.3174/ajnr.A3713 exclusion of gross structural abnormalities, but normally, actual disease mechanisms are not used as further information in a majority of these individuals. Voxel-based morphometry, DTI, and fMRI have been proposed as potential MR imaging biomarkers that might help overcome this shortcoming in the future. ${ }^{7,8}$

A prime drawback of $\mathrm{AMRI}$ is the rather high inter- and intraindividual variability of measures in conventional analyses, even in healthy individuals, ${ }^{14-16}$ that foils many such attempts. Conventional fMRI methods mainly comprise univariate activation or cofluctuation (functional-connectivity) analyses based on averaged signals in a few regions of interest or mass-univariate analyses across the whole brain, ${ }^{1}$ which come along with high requirements to control for multiple comparisons. ${ }^{17}$

\section{Overview of Machine-Learning-Based Classification Techniques for fMRI}

In the case of intertrial variability in individual subjects, the problem of differentiating single trials has been overcome in recent years by the rise of multivariate supervised learning methods derived from the fields of machine learning and pattern recognition. Such methods, often termed multivariate or multivoxel pattern analyses (MVPAs), are increasingly adopted in psychologically motivated fMRI studies. The concept of such analyses is that at first an algorithm is used to derive a decision rule (classifier) on the basis of a set of labeled training data (eg, comprising $\geq 2$ classes; eg, different stimuli categories or tasks). This rule is applied to classifying an independent set of test data as belonging to one of these classes in a second step. A general overview of this approach 


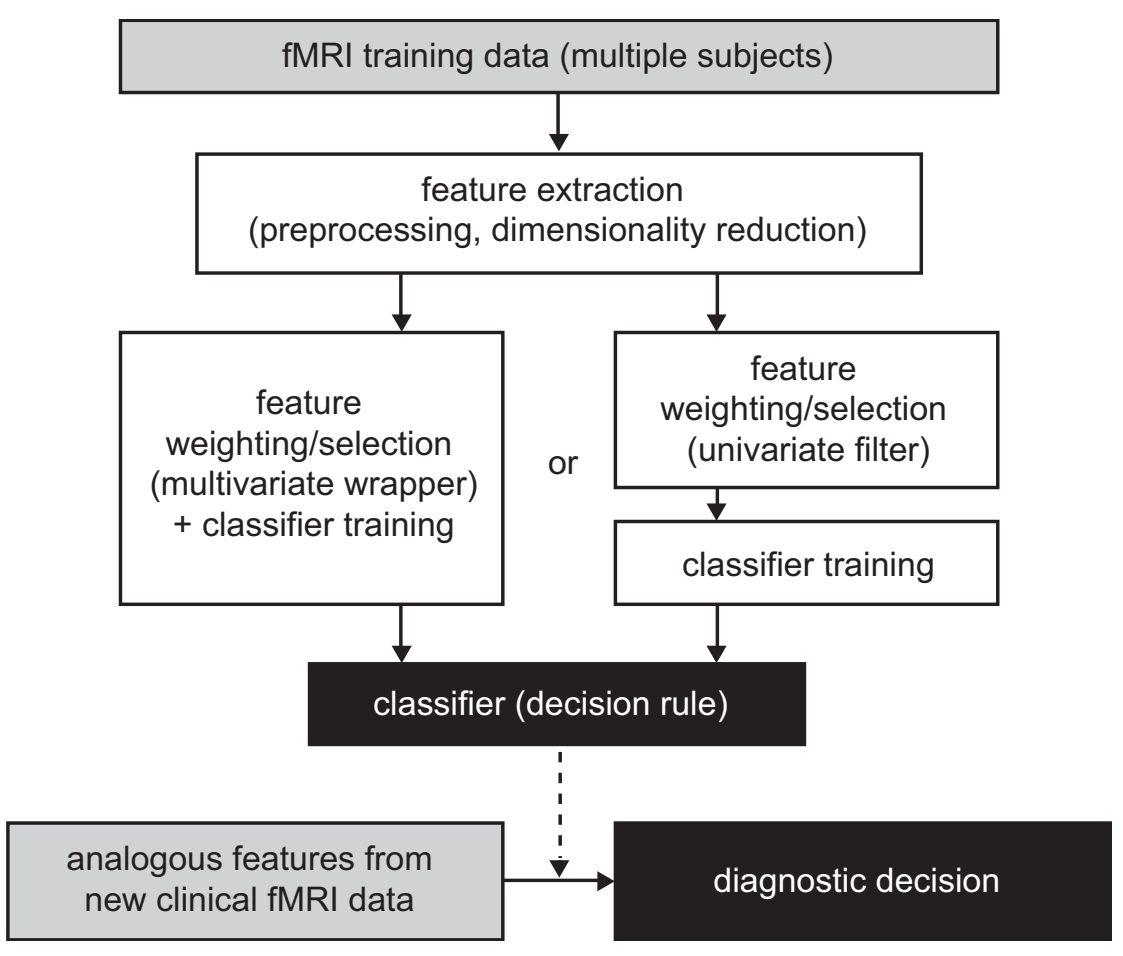

FIG 1. Illustration of the diagnostic workflow and data-processing pipeline proposed by most of the studies reviewed here.

is shown in Fig 1. In contrast to conventional analyses, these techniques are based on patterns of brain activation or connections not on individual regions or voxels. ${ }^{18-20}$ Recently this concept has been extended to classifying individual subjects with a diagnostic purpose (for earlier, methodologically oriented reviews see Kloppel et $\mathrm{al}^{21}$ and Orrù et $\mathrm{al}^{22}$ ). This article gives a comprehensive overview of MVPA applications to fMRI from a more clinical, particularly neuroradiologic, point of view.

Although there are a large number of supervised machinelearning techniques that can, in principle, be applied in this context, ${ }^{23} 2$ groups of methodologies are of particular importance: support vector machines (SVMs) and linear discriminant analyses (LDAs). In SVMs, the classification problem is operationalized as defining a hyperplane that best distinguishes groups of subjects. The classifier is trained by using a kernel by maximizing the margin of separation between 2 groups on the basis of the examples closest to the separating hyperplane. ${ }^{22-24}$ In a typical LDA variant, all data points are projected to a 1D space with the aim of maximizing intergroup separation and minimizing intraclass variation. ${ }^{22,23}$ LDA and support vector machines are very heterogeneous groups, depending on the actual operationalization or the kernel used. Certain kinds of SVMs are mathematically very similar to certain types of LDAs, while there can be important differences between different support vector machine formulations and parameter sets. ${ }^{23}$ The distinction made is, therefore, somewhat artificial.

fMRI datasets usually comprise several thousand nonindependent voxels. Yet the number of subjects is usually limited to dozens. This difference poses a certain problem for MVPA because most methods cannot deal with a high dimensionality of the data compared with the number of samples. There is a high risk of overfitting. This means that the classifier is perfectly trained to separate the samples used for training but has a poor ability to generalize to the successful classification of new data. This issue can be dealt with by the selection of classification methods that are less sensitive to a high dimensionality, such as SVMs. In contrast, LDA is usually very sensitive to this. Still, a strict dimensionality reduction is necessary: Primary data are preprocessed to concatenate redundant information by feature extraction, and features that are decisive are identified before actual classifier training by feature selection or weighting. Filter approaches, partially by using conventional univariate statistics or wrapper-based approaches, are commonly applied for feature selection. ${ }^{19}$

An issue that has to be overcome in diagnostic classification is interindividual structural variability regarding the morphology of the cerebral sulci and gyri as well as their relation to histologically and functionally relevant brain areas. ${ }^{25}$ Within-subject MVPA analyses often rely on fine-grained patterns on a single-voxel level. ${ }^{18,19}$ In contrast, most diagnostic MVPA studies reviewed here focus on another spatial scale: larger functionally coherent brain areas.

A specific feature present in the design of most MVPA-based fMRI studies is that datasets are often small and that classification performance is assessed through cross-validation (CV). Here, feature selection and classifier training are repeated several times. Each time a different range of datasets, often exactly one in the case of leave-one-out $\mathrm{CV}$, is excluded and used as a test set. ${ }^{19}$

\section{Recent Diagnostic fMRI Approaches Based on MVPA}

There has recently been a remarkable upsurge of scientific articles from the interdisciplinary functional neuroimaging community reporting successful applications of MVPA on fMRI data to various diagnostic problems, especially in the past 3 years. This constitutes a paradigm shift from comparative univariate to discriminative multivariate analyses of fMRI data. An exhaustive overview of these previous studies by using either task-based ${ }^{26-63}$ or task-free ${ }^{55,64-97} \mathrm{fMRI}$ is given in On-line Tables 1 and 2. An overview of particularly reliable studies with above-average statistical power is presented in Fig 2.

Although they are promising at first glance, there is a high degree of methodologic heterogeneity of classification algorithms and data-preprocessing steps in these studies. Some of the reported results seem to be mostly add-ons to studies whose designs were primarily aimed at clarifying disease mechanisms or were focused on computational aspects, not primarily done with the aim of developing a diagnostic tool. Until now, no single effort in this field has provided sufficient large-scale validation and systematic optimization of methodologic choices leading to an application in a real medical diagnostic setting. Due to this 




FIG 2. Overview of MVPA-based diagnostic fMRI studies with an above-average statistical power to detect successful models ( $n \geq 25$ in every group of the training set; group size ratio $\leq 1.2$ as a prerequisite for comparing the overall classification accuracies reported). ${ }^{34,43,45,55,61,72,74,85,95}$ Symbol sizes represent average group sizes in the respective study. a, Only minimum and maximum classification accuracies are shown here. b, Study of cross-validation findings also reports results in a smaller independent validation set. c, Only Gaussian process classifier overall accuracy is shown here. $\mathrm{d}$, High overlap with another study is not shown here. FS indicates feature selection. For a complete list of studies, see On-line Tables 1 and 2.

heterogeneity and because strategies to assess the statistical significance of diagnostic accuracy vary considerably between studies, we did not perform a formal meta-analytical comparison of these reports.

Data Acquisition and Preprocessing. By now a majority of reported approaches are based on conventional task-based fMRI. ${ }^{26-63}$ This means that patients have to perform a specific, mainly neuropsychological task in the MR imaging scanner. Statistical models are designed to evaluate the amount of variance in the acquired EPI data caused by this task modification of brain activity. This corresponds to "brain activation" in conventional fMRI studies. ${ }^{1}$ An advantage of this approach is a rather straightforward functional interpretability of such data. Yet in addition to mainly psychologically motivated studies in young healthy participants, patients' adherence to task instructions constitutes an important source of variability in real clinical settings and may even interfere with diagnostic decision-making.

Recently, a significant number of studies ${ }^{55,64-97}$ have been based on task-free fMRI acquisitions, so-called resting-state fMRI, which focuses on the functional connectivity of distant brain regions in terms of signal cofluctuations and therefore on the integrity of large-scale brain networks. ${ }^{98,99}$ A potential benefit of this method is that typical networks seem to be robustly identifiable in individual subjects. However, reports focusing on the reliability of typical resting-state fMRI measures highlight the problem that these are highly dependent on potentially confounding factors such as wakefulness or autonomic arousal. ${ }^{100,101}$ Although most resting-state fMRI findings in basic neuroscience are based on short acquisitions of approximately 5 minutes, which seem to be sufficient for network detection, ${ }^{102}$ there is recent evidence that retest reliability can be significantly improved by longer acquisitions. ${ }^{103}$ Only a minority of resting-state functional connectivity-based MVPA approaches have used acquisitions lasting at least 7 minutes. ${ }^{67,72,79,84,87,91}$

As a common analysis step on a single subject level, featureextraction methods are used to extract meaningful information from and simultaneously reduce the high dimensionality of the raw EPI time-series data. Prevailing approaches based on prior knowledge are activation modeling, based on general linear models for task-based acquisitions, ${ }^{1}$ and seed-to-voxel or region-of-interest to region-of-interest correlation analyses for task-free acquisitions. In addition, recently more complex graph-theoretic approaches have been derived from the ROI-based methods. Another way of analyzing task-based and task-free studies relies on data-driven approaches such as independent component analyses. ${ }^{98,104}$

Recent further developments in diagnostic MVPA are not solely based on one of these methods. For example, Du et $\mathrm{al}^{55}$ combined both task- and task-free fMRI in schizophrenia in a small study. Additionally, combinations of fMRI measures with volumetric data, ${ }^{41,48-50,63,76,78-81,86,89} \mathrm{DTI},{ }^{46,49,92}$ as well as genetics ${ }^{42}$ and behavioral data, ${ }^{40,41,50,76}$ have been used as features in MVPA analyses. However, results reported so far do not allow verified statements about the benefit of such multimodal acquisitions.

Feature Selection, Classifier Training, and Assessment of Classification Accuracy. Figure 2 and On-line Tables 1 and 2 contain information about the multivariate classification methods in the studies included in this review. They also contain information about whether the selection of potentially decisive features was 
based on conventional univariate analyses or whether it was also guided by multivariate information of distributed network patterns.

Apparently there are 2 groups of actual classification methods that have been applied successfully repeatedly: variants of LDA $^{26,53,59,61,65-67,70,73,79,81,82,94}$ and support vector machines. ${ }^{35,36,38,40-49,51,52,54,58,60,62,67-71,74-78,80,81,83-85,88-90,92,93,95-97}$ Although a certain number of articles report on conceptually different machine-learning techniques ${ }^{27-34,37,39,41,45,48,50,53-57,62,64,67,70,72,86-88,91}$ (eg, neural networks ${ }^{31,39,64,67,70}$ and decision tree-based approaches $^{41,50,81,86,87,91}$ ), each has only been applied occasionally, making reliable conclusions about their specific benefits and drawbacks in this context practically impossible.

With a few exceptions, ${ }^{72,75-78,80,81,86,88,89,95}$ the small sample sizes in most studies did not allow testing the classification accuracy in datasets completely independent of those used for classifier training. As stated above, a trick makes approximative assessments of classification accuracy of a set of trained classifiers possible: Most studies use CV to show the generalizability of strongly overlapping classifiers to new test data. This means that in most reports only the diagnostic ability of 1 particular set of dependent classifiers ${ }^{23}$ is proved. There is usually no formal test that allows conclusions regarding the ability of whole MVPA approaches (acquisition + feature extraction + feature selection + classifier training) to construct successful diagnostic tools in a particular clinical setting because $\mathrm{CV}$ is only used to assess classification of new data but not reliable classifier training independent from particular subjects. Additionally, setting up CV loops that do not strictly keep the test set and training set apart is a known source of error, leading to overoptimistic estimates of diagnostic accuracy. There is still some uncertainty regarding the most appropriate test of significance to be applied in the $\mathrm{CV}$ setting. ${ }^{19}$

Only a small subset of reports contains systematic comparisons and optimizations of larger sets of classification models used. ${ }^{49,68,70,75-78,80,81,86,88,89,95}$

\section{Potential Clinical Applications and Integration in Diagnostic Workflows}

To this point, most studies report applications to distinguish healthy controls and patients with a specific disease. These are a necessary step in developing and accessing diagnostic tools, but is it currently really clinically desirable to strive for such a tool?

In the context of practically illness-defining brain alterations, as in certain kinds of neurodegeneration, MVPA-fMRI methods might compete with radioactive tracer studies in the future. Regarding psychiatric diseases, it seems, for example, desirable to identify patients with a high risk of disease recurrence or progression. Especially in the case of major depressive disorders, there are a number of patients who do not respond to standard pharmacologic treatment; this outcome hints at potentially underlying divergent biologic mechanisms. Prediction of treatment response to a certain group of drugs seems to be a valuable objective as well. ${ }^{9}$ To date, some MVPA-fMRI studies have already attempted to classify subjects regarding prognostically relevant subgroups. ${ }^{36,38,40,46,49,50,52,53,56,58}$ Another important but overlapping clinical question may be how to distinguish patients with neurobiologically different disease entities but with a similar initial clinical presentation such as unipolar and bipolar depression. Such differential diagnostic aspects have been addressed in a few recent MVPA-fMRI studies as well. ${ }^{32,39,51,54,57,71,73,75,78,80,81,88,89,94,95}$

In this context, specific features of most psychiatric diseases should be taken into account when discussing the results of these analyses: The etiology and progression of disease are complex and only partly attributable to biologic causes. The biopsychosocial model of pathogenesis includes major influences of social and life event-related factors ${ }^{105,106}$ that do not necessarily lead to correlates that are approachable by biologic measures such as fMRI. ${ }^{107}$ Furthermore, many diagnostically relevant symptoms are, by definition, subjective (eg, depressed mood). ${ }^{108}$ The burden of suffering is often decisive in terms of indications for treatment. ${ }^{109}$ Therefore, fMRI-MVPA-based measures should not be expected to become the criterion standard in diagnostics and replace indepth history-taking. The accuracies of the studies reviewed here support this theoretic argument. Still, imaging-based multivariate tools might be able to provide clinically useful additional information: When important information (eg, regarding prognosis) is, by definition, not deducible from the course of disease, these tools might provide the clinician with crucial hints, ${ }^{7}$ unraveling the "biologic share" of disease.

In nearly all fMRI-MVPA studies, there was a significant amount of misclassifications (On-line Tables 1 and 2). Partially, they may be attributable to inherent noise in the data and remaining methodologic weaknesses in data analysis. However, misclassification might also be based on biologically and medically meaningful information like the effects of medication ${ }^{110}$ and age. ${ }^{69,85}$ Sex effects are a much-debated issue in fMRI as well. ${ }^{111,112}$ Further investigation of misclassified subjects might even pose a starting point to identify biologically different disease subgroups. Supposedly, a practical problem is that the referring physician and the radiologist cannot easily grasp what leads to a single diagnostic decision by fMRI-MVPA. In comparison with other types of diagnostic imaging, it is therefore not directly possible to appreciate the extent of potentially biasing features in a specific subject. Only 5 recent studies have tried to overcome this issue by introducing individual confidence measures. ${ }^{39,48,54,56,57}$

As seen in Fig 2 and On-line Table 1, MVPA-fMRI has already been applied to a larger number of psychiatric disease entities. For depression, ${ }^{35,36,38,45,47,48,54,57,68,84}$ schizophrenia, ${ }^{26,32-34,37,39,42-44,55,58,61,62,64,66,70,74,90,91,96}$ and Alzheimer dementia, ${ }^{26,27,41,50,53,65,71,73,79,82,94}$ there is now a larger body of independent work.

The diversity of scientific backgrounds of recent studies is reflected by a striking heterogeneity of reported methodologic details, sample characteristics, validation strategies, and performance measures. This heterogeneity limits effort to draw more reliable quantitative conclusions about the clinical benefits of MVPA-fMRI at this stage. More specifically designed studies with a sufficiently high statistical power and confounding factors of a real clinical setting in mind with a more standardized diagnostic end point should be performed to facilitate meta-analytic comparisons in the future. As a stimulus for further debate, we propose reporting standards and standards of study design that, in our opinion, may help overcome some of these issues. They are 
Proposed reporting standards for future studies applying multivariate classification techniques to fMRI data with a diagnostic intention in addition to general reporting standards in fMRI research

Proposed Reporting Standards Desirable Aspects of Study Design

Report of overall classification accuracies/generalization rate

(in addition to sensitivity and specificity if applicable)

Rigorous statistical tests

Report of all tested models (not only optimized/best models) Clearly identify inferential tests and explorative analyses

Clearly identify feature extraction and feature selection/weighting strategies

Denominate origin of classification algorithms if an independent toolbox is used for pipeline building

Good clinical characterization of the sample including demographic data (psychometric data if applicable)
Sufficiently large, equally sized groups (25 subjects per group desirable, but depending on specific methodologies used and power analyses)

Sufficiently long scan duration for resting state fMRI (at least 5 minutes, evaluation of a potential benefit of longer acquisitions desirable)

Systematic model optimization

Well-chosen and well-defined clinical problem

Multisite/multiscanner studies

Evaluation of functionally/anatomically interpretable classifiers and methods with individual reliability measures

Independent validation set summarized in the Table. Before MVPA-fMRI could be applied in real clinical settings, potential interscanner variability ${ }^{113}$ should also be taken into account.

\section{CONCLUSIONS}

Approximately 70 studies at the proof-of-principle level that use MVPA of fMRI data with a diagnostic intention have been reported. However, there is wide range of different methodologic decisions, from data-acquisition strategies through preprocessing and feature selection to actual diagnostic classification algorithms and parameter settings and, therefore, a high flexibility in study design. Results reported as yet are mainly based on small sets of subjects. Therefore, one has to be cautious in drawing reliable conclusions on the basis of this literature. Published results may just represent the tip of the iceberg, with a lot more unsuccessful unpublished attempts to apply this methodology. Therefore, there might be an important publication bias, and published results regarding the statistical significance of successful diagnostic classification should be interpreted in the light of a potential need to correct for multiple comparisons. ${ }^{114}$ Nevertheless, it can be regarded meanwhile as an independently replicated finding that building on task-based and resting-state fMRI as well support vector machines as LDA approaches has the potential to differentiate patients from healthy subjects in psychiatric disorders with most repeated findings in dementia, schizophrenia, and depression.

In contrast, there is apparently more uncertainty regarding optimal strategies for data preprocessing and feature selection, advisable steps to allow the classification algorithm to work despite a very high dimensionality and noise level of the original data. Many of these methods are derived from conventional fMRI analysis methods. Hardly any effort seems to have been made to systematically compare and evaluate the influence of these different approaches and parameter-setting selections on diagnostic accuracy.

In conclusion, here is some evidence that MVPA-fMRI is promising for overcoming long-known reliability issues in fMRI and providing clinically important prognostic and differential diagnostic information in psychiatric disorders beyond pure exclusion of gross structural alterations. Despite the optimism coming from the recent discussion in the interdisciplinary functional neu- roimaging community, this method is still rather new, and work has to be done to validate methodologic choices and identify those specific clinical settings that really allow a beneficial application. Moreover, a conceivable integration of MVPA-based fMRI into clinical workflow will depend critically on tackling diagnostic problems with a real clinical benefit and effects on therapeutic decision-making.

Disclosures: Wolfram Schwindt-UNRELATED: payment for manuscript preparation: RöFo (Executive Editor).

\section{REFERENCES}

1. Huettel SA, Song AW, McCarthy G. Functional Magnetic Resonance Imaging. Sunderland, Massachusetts: Sinauer Associates; 2009

2. Zhang D, Raichle ME. Disease and the brain's dark energy. Nat Rev Neurol 2010;6:15-28

3. Chen $\mathrm{CH}$, Suckling J, Lennox BR, et al. A quantitative meta-analysis of fMRI studies in bipolar disorder. Bipolar Disord 2011;13:1-15

4. Bartsch AJ, Homola G, Biller A, et al. Diagnostic functional MRI: illustrated clinical applications and decision-making. J Magn Reson Imaging 2006;23:921-32

5. Pillai JJ. The evolution of clinical functional imaging during the past 2 decades and its current impact on neurosurgical planning. AJNR Am J Neuroradiol 2010;31:219-25

6. Gruber O. Neuroimaging markers: their role for differential diagnosis and therapeutic decisions in personalized psychiatry [in German]. Nervenarzt 2011;82:1404, 406, 408

7. Linden DE. The challenges and promise of neuroimaging in psychiatry. Neuron 2012;73:8-22

8. Oertel-Knöchel V, Bittner RA, Knochel C, et al. Discovery and development of integrative biological markers for schizophrenia. Prog Neurobiol 2011;95:686-702

9. Mössner R, Mikova O, Koutsilieri E, et al. Consensus paper of the WFSBP task force on biological markers: biological markers in depression. World J Biol Psychiatry 2007;8:141-74

10. Hampel H, Lista S, Khachaturian ZS. Development of biomarkers to chart all Alzheimer's disease stages: the royal road to cutting the therapeutic gordian knot. Alzheimers Dement 2012;8:312-36

11. Hyman SE. Can neuroscience be integrated into the DSM-V? Nat Rev Neurosci 2007;8:725-32

12. de Souza LC, Lehericy S, Dubois B, et al. Neuroimaging in dementias. Curr Opin Psychiatry 2012;25:473-79

13. Jack CR, Jr. Alzheimer disease: new concepts on its neurobiology and the clinical role imaging will play. Radiology 2012;263:344-61

14. Mueller S, Wang D, Fox MD, et al. Individual variability in func- 
tional connectivity architecture of the human brain. Neuron 2013;77:586-95

15. Raemaekers M, du Plessis S, Ramsey NF, et al. Test-retest variability underlying fMRI measurements. Neuroimage 2012;60:717-27

16. Shehzad Z, Kelly AM, Reiss PT, et al. The resting brain: unconstrained yet reliable. Cereb Cortex 2009;19:2209-29

17. Nichols TE. Multiple testing corrections, nonparametric methods, and random field theory. Neuroimage 2012;62:811-15

18. Norman KA, Polyn SM, Detre GJ, et al. Beyond mind-reading: multi-voxel pattern analysis of fMRI data. Trends Cogn Sci 2006;10:424-30

19. Pereira F, Mitchell T, Botvinick M. Machine learning classifiers and fMRI: a tutorial overview. Neuroimage 2009;45:S199-209

20. Haynes JD, Rees G. Decoding mental states from brain activity in humans. Nat Rev Neurosci 2006;7:523-34

21. Klöppel S, Abdulkadir A, Jack CR Jr, et al. Diagnostic neuroimaging across diseases. Neuroimage 2012;61:457-63

22. Orrù G, Pettersson-Yeo W, Marquand AF, et al. Using support vector machine to identify imaging biomarkers of neurological and psychiatric disease: a critical review. Neurosci Biobehav Rev 2012;36:1140-52

23. Alpaydin E. Introduction to Machine Learning. Cambridge, Massachusetts: MIT Press; 2010

24. Vapnik VN. The Nature of Statistical Learning Theory. 2nd ed. New York: Springer-Verlag; 2000

25. Derrfuss J, Brass M, von Cramon DY, et al. Neural activations at the junction of the inferior frontal sulcus and the inferior precentral sulcus: interindividual variability, reliability, and association with sulcal morphology. Hum Brain Mapp 2009;30:299-311

26. Ford J, Farid H, Makedon F, et al. Patient classification of fMRI activation maps. In: Ellis R, Peters T, eds. Medical Image Computing and Computer-Assisted Intervention - MICCAI 2003, Lecture Notes in Computer Science, Vol. 2879. Berlin, Germany: Springer-Verlag; 2003:58

27. Kontos D, Megalooikonomou V, Pokrajac D, et al. Extraction of discriminative functional MRI activation patterns and an application to Alzheimer's disease. In: Barillot C, Haynor DR, Hellier P, eds. Medical Image Computing and Computer-Assisted Intervention MICCAI 2004, Lecture Notes in Computer Science, Vol. 3217. Berlin, Germany: Springer-Verlag. 2004;727-35

28. Bogorodzki P, Rogowska J, Yurgelun-Todd DA. Structural group classification technique based on regional $\mathrm{AMRI}$ BOLD responses. IEEE Trans Med Imaging 2005;24:389-98

29. Zhang L, Samaras D, Tomasi D, et al. Exploiting temporal information in functional magnetic resonance imaging brain data. Med Image Comput Comput Assist Interv 2005;8:679-87

30. Shinkareva SV, Ombao HC, Sutton BP, et al. Classification of functional brain images with a spatio-temporal dissimilarity map. $\mathrm{Neu}$ roimage 2006;33:63-71

31. Chen R, Herskovits EH. Clinical diagnosis based on bayesian classification of functional magnetic-resonance data. Neuroinformatics 2007;5:178-88

32. Calhoun VD, Maciejewski PK, Pearlson GD, et al. Temporal lobe and "default" hemodynamic brain modes discriminate between schizophrenia and bipolar disorder. Hum Brain Mapp 2008;29:1265-75

33. Demirci O, Clark VP, Calhoun VD. A projection pursuit algorithm to classify individuals using fMRI data: application to schizophrenia. Neuroimage 2008;39:1774-82

34. Demirci O, Clark VP, Magnotta VA, et al. A review of challenges in the use of fMRI for disease classification/characterization and a projection pursuit application from multi-site fMRI schizophrenia study. Brain Imaging Behav 2008;2:147-226

35. Fu CH, Mourão-Miranda J, Costafreda SG, et al. Pattern classification of sad facial processing: toward the development of neurobiological markers in depression. Biol Psychiatry 2008;63:656-62

36. Marquand AF, Mourão-Miranda J, Brammer MJ, et al. Neuroanatomy of verbal working memory as a diagnostic biomarker for depression. Neuroreport 2008;19:1507-11
37. Michael AM, Calhoun VD, Andreasen NC, et al. A method to classify schizophrenia using inter-task spatial correlations of functional brain images. Conf Proc IEEE Eng Med Biol Soc 2008;2008:5510-13

38. Costafreda SG, Khanna A, Mourão-Miranda J, et al. Neural correlates of sad faces predict clinical remission to cognitive behavioural therapy in depression. Neuroreport 2009;20:637-41

39. Arribas JI, Calhoun VD, Adali T. Automatic bayesian classification of healthy controls, bipolar disorder, and schizophrenia using intrinsic connectivity maps from fMRI data. IEEE Trans Biomed Eng 2010;57:2850-60

40. Saur D, Ronneberger O, Kummerer D, et al. Early functional magnetic resonance imaging activations predict language outcome after stroke. Brain 2010;133:1252-64

41. Tripoliti EE, Fotiadis DI, Argyropoulou M, et al. A six stage approach for the diagnosis of the Alzheimer's disease based on fMRI data. J Biomed Inform 2010;43:307-20

42. Yang H, Liu J, Sui J, et al. A hybrid machine learning method for fusing fMRI and genetic data: combining both improves classification of schizophrenia. Front Hum Neurosci 2010;4:192

43. Castro E, Martinez-Ramon M, Pearlson G, et al. Characterization of groups using composite kernels and multi-source fMRI analysis data: application to schizophrenia. Neuroimage 2011;58:526-36

44. Costafreda SG, Fu CH, Picchioni M, et al. Pattern of neural responses to verbal fluency shows diagnostic specificity for schizophrenia and bipolar disorder. BMC Psychiatry 2011;11:18

45. Hahn T, Marquand AF, Ehlis AC, et al. Integrating neurobiological markers of depression. Arch Gen Psychiatry 2011;68:361-68

46. Hoeft F, McCandliss BD, Black JM, et al. Neural systems predicting long-term outcome in dyslexia. Proc Natl Acad Sci U $S$ A 2011;108:361-66

47. Mourão-Miranda J, Hardoon DR, Hahn T, et al. Patient classification as an outlier detection problem: an application of the one-class support vector machine. Neuroimage 2011;58:793-804

48. Nouretdinov I, Costafreda SG, Gammerman A, et al. Machine learning classification with confidence: application of transductive conformal predictors to MRI-based diagnostic and prognostic markers in depression. Neuroimage 2011;56:809-13

49. Rizk-Jackson A, Stoffers D, Sheldon S, et al. Evaluating imaging biomarkers for neurodegeneration in pre-symptomatic Huntington's disease using machine learning techniques. Neuroimage 2011;56:788-96

50. Tripoliti EE, Fotiadis DI, Argyropoulou M. A supervised method to assist the diagnosis and monitor progression of Alzheimer's disease using data from an fMRI experiment. Artif Intell Med 2011;53:35-45

51. Weygandt M, Schaefer A, Schienle A, et al. Diagnosing different binge-eating disorders based on reward-related brain activation patterns. Hum Brain Mapp 2012;33:2135-46

52. Abdulkadir A, Ronneberger O, Wolf RC, et al. Functional and structural MRI biomarkers to detect pre-clinical neurodegeneration. Curr Alzheimer Res 2013;10:125-34

53. Andersen AH, Rayens WS, Liu Y, et al. Partial least squares for discrimination in fMRI data. Magn Reson Imaging 2012;30:446-52

54. Grotegerd D, Suslow T, Bauer J, et al. Discriminating unipolar and bipolar depression by means of fMRI and pattern classification: a pilot study. Eur Arch Psychiatry Clin Neurosci 2013;63:119-31

55. Du W, Calhoun VD, Li H, et al. High classification accuracy for schizophrenia with rest and task fMRI data. Front Hum Neurosci 2012;6:145

56. Mourão-Miranda J, Oliveira L, Ladouceur CD, et al. Pattern recog nition and functional neuroimaging help to discriminate healthy adolescents at risk for mood disorders from low risk adolescents. PLoS One 2012;7:e29482

57. Mourão-Miranda J, Almeida JR, Hassel S, et al. Pattern recognition analyses of brain activation elicited by happy and neutral faces in unipolar and bipolar depression. Bipolar Disord 2012;14:451-60

58. Nejad AB, Madsen KH, Ebdrup BH, et al. Neural markers of negative symptom outcomes in distributed working memory brain activity 
of antipsychotic-naive schizophrenia patients. Int J Neuropsychopharmacol 2013;16:1195-204

59. Ramezani M, Abolmaesumi $\mathrm{P}$, Marble $\mathrm{K}$, et al. Classification of individuals based on sparse representation of brain cognitive patterns: a functional MRI study. Conf Proc IEEE Eng Med Biol Soc 2012;2012:2688-91

60. Weygandt M, Blecker CR, Schafer A, et al. fMRI pattern recognition in obsessive-compulsive disorder. Neuroimage 2012;60:1186-93

61. Yoon JH, Nguyen DV, McVay LM, et al. Automated classification of fMRI during cognitive control identifies more severely disorganized subjects with schizophrenia. Schizophr Res 2012;135:28-33

62. Rish I, Cecchi G, Thyreau B, et al. Schizophrenia as a network disease: disruption of emergent brain function in patients with auditory hallucinations. PLoS One 2013;8:e50625

63. Kim J, Lee JH. Integration of structural and functional magnetic resonance imaging improves mild cognitive impairment detection. Magn Reson Imaging 2013;31:718-32

64. Jafri MJ, Calhoun VD. Functional classification of schizophrenia using feed forward neural networks. Conf Proc IEEE Eng Med Biol Soc 2006; (suppl):6631-34

65. Wang $\mathrm{K}$, Jiang $\mathrm{T}$, Liang $\mathrm{M}$, et al. Discriminative analysis of early Alzheimer's disease based on two intrinsically anti-correlated networks with resting-state fMRI. Med Image Comput Comput Assist Interv 2006;9:340-47

66. Shi F, Liu Y, Jiang $\mathrm{T}$, et al. Regional homogeneity and anatomical parcellation for fMRI image classification: application to schizophrenia and normal controls. Med Image Comput Comput Assist Interv 2007;10(pt 2):136-43

67. Zhu CZ, Zang YF, Cao QJ, et al. Fisher discriminative analysis of resting-state brain function for attention-deficit/hyperactivity disorder. Neuroimage 2008;40:110-20

68. Craddock RC, Holtzheimer PE 3rd, Hu XP, et al. Disease state prediction from resting state functional connectivity. Magn Reson Med 2009;62:1619-28

69. Supekar K, Musen M, Menon V. Development of large-scale functional brain networks in children. PLoS Biol 2009;7:e1000157

70. Shen $\mathrm{H}$, Wang L, Liu Y, et al. Discriminative analysis of resting-state functional connectivity patterns of schizophrenia using low dimensional embedding of fMRI. Neuroimage 2010;49:3110-21

71. Zhou J, Greicius MD, Gennatas ED, et al. Divergent network connectivity changes in behavioural variant frontotemporal dementia and Alzheimer's disease. Brain 2010;133:1352-67

72. Anderson JS, Nielsen JA, Froehlich AL, et al. Functional connectivity magnetic resonance imaging classification of autism. Brain 2011;134:3742-54

73. Chen G, Ward BD, Xie C, et al. Classification of Alzheimer disease, mild cognitive impairment, and normal cognitive status with largescale network analysis based on resting-state functional MR imaging. Radiology 2011;259:213-21

74. Fan $\mathrm{Y}$, Liu $\mathrm{Y}, \mathrm{Wu} \mathrm{H}$, et al. Discriminant analysis of functional connectivity patterns on Grassmann manifold. Neuroimage 2011;56:2058-67

75. Brown MR, Sidhu GS, Greiner R, et al. ADHD-200 global competition: Diagnosing ADHD using personal characteristic data can outperform resting state fMRI measurements. Front Syst Neurosci 2012;6:69

76. Bohland JW, Saperstein S, Pereira F, et al. Network, anatomical, and non-imaging measures for the prediction of ADHD diagnosis in individual subjects. Front Syst Neurosci 2012;6:78

77. Cheng W, Ji X, Zhang J, et al. Individual classification of ADHD patients by integrating multiscale neuroimaging markers and advanced pattern recognition techniques. Front Syst Neurosci 2012;6:58

78. Colby JB, Rudie JD, Brown JA, et al. Insights into multimodal imaging classification of ADHD. Front Syst Neurosci 2012;6:59

79. Dai Z, Yan C, Wang Z, et al. Discriminative analysis of early Alzheimer's disease using multi-modal imaging and multi-level characterization with multi-classifier (M3). Neuroimage 2012;59:2187-95
80. Dai D, Wang J, Hua J, et al. Classification of ADHD children through multimodal magnetic resonance imaging. Front Syst Neurosci 2012;6:63

81. Eloyan A, Muschelli J, Nebel MB, et al. Automated diagnoses of attention deficit hyperactive disorder using magnetic resonance imaging. Front Syst Neurosci 2012;6:61

82. Koch W, Teipel S, Mueller S, et al. Diagnostic power of default mode network resting state $\mathrm{FMRI}$ in the detection of Alzheimer's disease. Neurobiol Aging 2012;33:466-78

83. Long D, Wang J, Xuan M, et al. Automatic classification of early Parkinson's disease with multi-modal MR imaging. PLoS One 2012;7:e47714

84. Ma Q, Zeng LL, Shen $\mathrm{H}$, et al. Altered cerebellar-cerebral restingstate functional connectivity reliably identifies major depressive disorder. Brain Res 2013;1495:86-94

85. Meier TB, Desphande AS, Vergun S, et al. Support vector machine classification and characterization of age-related reorganization of functional brain networks. Neuroimage 2012;60:601-13

86. Olivetti E, Greiner S, Avesani P. ADHD diagnosis from multiple data sources with batch effects. Front Syst Neurosci 2012;6:70

87. Richiardi J, Gschwind M, Simioni S, et al. Classifying minimally disabled multiple sclerosis patients from resting state functional connectivity. Neuroimage 2012;62:2021-33

88. Sato JR, Hoexter MQ, Fujita A, et al. Evaluation of pattern recognition and feature extraction methods in ADHD prediction. Front Syst Neurosci 2012;6:68

89. Sidhu GS, Asgarian N, Greiner R, et al. Kernel principal component analysis for dimensionality reduction in fMRI-based diagnosis of ADHD. Front Syst Neurosci 2012;6:74

90. Tang $\mathrm{Y}$, Wang L, Cao F, et al. Identify schizophrenia using restingstate functional connectivity: an exploratory research and analysis. Biomed Eng Online 2012;11:50

91. Venkataraman A, Whitford TJ, Westin CF, et al. Whole brain resting state functional connectivity abnormalities in schizophrenia. Schizophr Res 2012;139:7-12

92. Wee CY, Yap PT, Zhang D, et al. Identification of MCI individuals using structural and functional connectivity networks. Neuroimage 2012;59:2045-56

93. Zeng LL, Shen $\mathrm{H}$, Liu L, et al. Identifying major depression using whole-brain functional connectivity: a multivariate pattern analysis. Brain 2012;135:1498-507

94. Zhang Z, Liu Y, Jiang T, et al. Altered spontaneous activity in Alzheimer's disease and mild cognitive impairment revealed by regional homogeneity. Neuroimage 2012;59:1429-40

95. Fair DA, Nigg JT, Iyer S, et al. Distinct neural signatures detected for ADHD subtypes after controlling for micro-movements in resting state functional connectivity MRI data. Front Syst Neurosci 2012;6:80

96. Yu Y, Shen H, Zhang H, et al. Functional connectivity-based signatures of schizophrenia revealed by multiclass pattern analysis of resting-state fMRI from schizophrenic patients and their healthy siblings. Biomed Eng Online 2013;12:10

97. Zhang D, Liu B, Chen J, et al. Determination of vascular dementia brain in distinct frequency bands with whole brain functional connectivity patterns. PLoS One 2013;8:e54512

98. van den Heuvel MP, Hulshoff Pol HE. Exploring the brain network: a review on resting-state fMRI functional connectivity. Eur Neuropsychopharmacol 2010;20:519-34

99. Lee MH, Smyser CD, Shimony JS. Resting-state fMRI: a review of methods and clinical applications. AJNR Am J Neuroradiol 2013;34: $1866-72$

100. Fan J, Xu P, Van Dam NT, et al. Spontaneous brain activity relates to autonomic arousal. J Neurosci 2012;32:11176-86

101. De Havas JA, Parimal S, Soon CS, et al. Sleep deprivation reduces default mode network connectivity and anti-correlation during rest and task performance. Neuroimage 2012;59:1745-51

102. Van Dijk KR, Hedden T, Venkataraman A, et al. Intrinsic func- 
tional connectivity as a tool for human connectomics: theory, properties, and optimization. J Neurophysiol 2010;103:297-321

103. Anderson JS, Ferguson MA, Lopez-Larson M, et al. Reproducibility of single-subject functional connectivity measurements. AJNR Am J Neuroradiol 2011;32:548-55

104. Margulies DS, Bottger J, Long X, et al. Resting developments: a review of fMRI post-processing methodologies for spontaneous brain activity. MAGMA 2010;23:289-307

105. Adler RH. Engel's biopsychosocial model is still relevant today. J Psychosom Res 2009;67:607-11

106. Engel GL. The need for a new medical model: a challenge for biomedicine. Science 1977;196:129-36

107. Smith RC, Fortin AH, Dwamena F, et al. An evidence-based patient-centered method makes the biopsychosocial model scientific. Patient Educ Couns 2013;91:265-70

108. Fuchs T. Subjectivity and intersubjectivity in psychiatric diagnosis. Psychopathology 2010;43:268-74
109. Büchi S, Sensky T, Sharpe L, et al. Graphic representation of illness: a novel method of measuring patients' perceptions of the impact of illness. Psychother Psychosom 1998;67:222-25

110. Marquand AF, De Simoni S, O’Daly OG, et al. Pattern classification of working memory networks reveals differential effects of methylphenidate, atomoxetine, and placebo in healthy volunteers. Neuropsychopharmacology 2011;36:1237-47

111. Biswal BB, Mennes M, Zuo XN, et al. Toward discovery science of human brain function. Proc Natl Acad Sci U S A 2010;107:4734-39

112. Gong G, He Y, Evans AC. Brain connectivity: gender makes a difference. Neuroscientist 2011;17:575-91

113. Friedman L, Glover GH, Krenz D, et al. Reducing inter-scanner variability of activation in a multicenter fMRI study: role of smoothness equalization. Neuroimage 2006;32:1656-68

114. Ioannidis JP. Why most published research findings are false. PLoS Med 2005;2:e124 